\title{
Infecção pelo SARS-CoV-2 e Tromboembolismo Pulmonar: Importância do diagnóstico por imagem
}

\author{
SARS-CoV-2 infection and pulmonary thromboembolism: Importance of diagnostic imaging \\ Infección por SARS-CoV-2 y Tromboembolismo Pulmonar: Importancia del diagnóstico por \\ imagen
}

Recebido: 12/04/2021 | Revisado: 19/04/2021 | Aceito: 24/04/2021 | Publicado: 09/05/2021

\author{
Débora Silva Amorim \\ ORCID: https://orcid.org/0000-0003-0894-9635 \\ Unidade de Ensino Superior de Feira de Santana, Brasil \\ E-mail: amorimdebora_@outlook.com \\ João Lúcio Macário Lira \\ ORCID: https://orcid.org/0000-0002-0043-6125 \\ Centro Universitário CESMAC, Brasil \\ E-mail: joaoluciomacario@gmail.com \\ Rafaela da Silva Moreira \\ ORCID: https://orcid.org/0000-0002-7772-1397 \\ Unidade de Ensino Superior de Feira de Santana, Brasil \\ E-mail: rafaelamoreira_smoreira@hotmail.com \\ Flávia Nunes Fernandes \\ ORCID: https://orcid.org/0000-0002-0436-0797 \\ Unidade de Ensino Superior de Feira de Santana, Brasil \\ E-mail: fernandesnflavia@gmail.com \\ Roberta Amorim de Matos \\ ORCID: https://orcid.org/0000-0002-4655-4290 \\ Unidade de Ensino Superior de Feira de Santana, Brasil \\ E-mail: robertaamorim9@hotmail.com \\ Maríllia Ferreira Calado \\ ORCID: https://orcid.org/0000-0002-5321-9351 \\ Faculdade Maurício de Nassau Caruaru, Brasil \\ E-mail: marillia_calado@hotmail.com \\ Felicson Leonardo Oliveira Lima \\ ORCID: https://orcid.org/0000-0001-5256-6768 \\ Faculdade Nobre de Feira de Santana, Brasil \\ E-mail: felicsonleonardo@hotmail.com, Brasil \\ Carlos Danilo Cardoso Matos Silva \\ ORCID: https://orcid.org/0000-0001-6536-724X \\ Unidade de Ensino Superior de Feira de Santana, Brasil \\ E-mail: carloscardoso.fsa@gmail.com
}

\begin{abstract}
Resumo
Objetivo: Apresentar a relação da infecção pelo SARS-CoV-2 com o tromboembolismo pulmonar, evidenciando assim, a importância dos exames de imagem no auxílio do diagnóstico desta patologia, enfatizando, as principais técnicas utilizadas, tais como: Radiografia, Tomografia Computadorizada e Angiografia Pulmonar. Metodologia: O presente estudo trata-se de uma revisão integrativa de literatura de caráter descritiva e qualitativa, nas bases de dados eletrônicas Centers for Disease Control and Prevention (CDC), Scientific Electronic Library Online (SciELO), PubMed (MEDLINE), foram incluídos artigos publicados exclusivamente no ano de 2020. Resultados e Discussão: A COVID-19 é uma infecção viral respiratória que pode evoluir para doença trombótica ou formação de tromboembolismo, pois está associada ao aumento de quimiocinas e citocinas pró-inflamatórias, que levam ao aumento da permeabilidade vascular, entre outros. Devido a incidência de doenças trombóticas em pacientes acometidos pelo novo coronavírus, tem-se utilizado os exames de imagem, dentre eles, o Raio-X e TC de tórax apresentam melhores resultados evidentes. Contudo, a TC apresenta especificidade e sensibilidade superior se comparada a Radiografia. Conclusão: Concluiu-se que a COVID-19 provoca alterações hematológicas que predispõem a formação de tromboembolismo, diante dessas alterações faz-se necessário a utilização dos exames por imagem, sendo eles imprescindíveis para a confirmação do diagnóstico.
\end{abstract}

Palavras-chave: Infecções por coronavírus; Tromboembolismo; Exame por imagem; COVID-19. 


\begin{abstract}
Objective: To present the relationship of SARS-CoV-2 hair infection as pulmonary thromboembolism, also evidencing, at the same time, two non-auxiliary imaging tests for the diagnosis of this pathology, emphasizing the main techniques used, such as: Radiography, Computerized Tomography and Angiography Pulmonary. Methodology: $\mathrm{O}$ present study is about an integrative review of descriptive and qualitative literature, based on electronic databases Centers for Disease Control and Prevention (CDC), Scientific Electronic Library Online (SciELO), PubMed (MEDLINE), included foram Articles published exclusively in the year 2020. Results and Discussion: COVID-19 is a respiratory viral infection that can evolve to cause thrombotic disease or formation of thromboembolism, it is associated with an increase in chemokines and pro-inflammatory cytokines, which leads to an increase in vascular permeability, among others. Due to the incidence of thrombotic diseases in patients with novo coronavirus, imaging exams were used, within them, or X-ray and chest CT, showing less obvious results. Contudo, CT shows higher specificity and sensitivity compared to X-ray. Conclusion: It is concluded that COVID-19 causes haematological alterations that predispose to the formation of thromboembolism, due to its facial alterations it is necessary to use two examinations per image, being essential for a confirmation of diagnosis.
\end{abstract}

Keywords: Coronavirus infections; Thromboembolism; Examination by Image; COVID-19.

\begin{abstract}
Resumen
Objetivo: Presentar la relación entre la infección por SARS-CoV-2 y el tromboembolismo pulmonar, evidenciando así la importancia de las pruebas de imagen para ayudar en el diagnóstico de esta patología, destacando las principales técnicas utilizadas, tales como: Radiografía, Tomografía Computada y Angiografía Pulmonar. Metodología: El presente estudio es una revisión integradora de la literatura descriptiva y cualitativa, en las bases de datos electrónicas Centers for Disease Control and Prevention (CDC), Scientific Electronic Library Online (SciELO), PubMed (MEDLINE), se incluyeron artículos publicados exclusivamente en el año 2020. Resultados y Discusión: COVID-19 es una infección viral respiratoria que puede progresar a enfermedad trombótica o formación de tromboembolismo, ya que se asocia a un aumento de quimiocinas y citocinas proinflamatorias, que conducen a una mayor permeabilidad vascular, entre otras. Debido a la incidencia de enfermedades trombóticas en pacientes afectados por el nuevo coronavirus, se han utilizado pruebas de imagen, entre ellas, la radiografía y la tomografía computarizada de tórax tienen mejores resultados evidentes. Sin embargo, la TC tiene una especificidad y una sensibilidad superiores en comparación con la radiografía. Conclusión: Se concluyó que el COVID-19 ocasiona cambios hematológicos que predisponen a la formación de tromboembolismo, ante estos cambios es necesario el uso de pruebas de imagen, las cuales son fundamentales para la confirmación diagnóstica.
\end{abstract}

Palabras clave: Infecciones por coronavirus; Tromboembolismo; Examen por imagen; COVID-19.

\title{
1. Introdução
}

Severe Acute Respiratory Syndrome Coronavirus 2 (SARS-CoV-2) é um vírus com registros iniciais em dezembro de 2019, em Wuhan, na China. O mesmo é responsável por causar a Coronavirus Disease 2019 (COVID-19), a qual é uma doença que acomete o trato respiratório inferior e superior (Cespedes; Souza, 2020). A COVID-19 suscita sintomas que vão desde um simples resfriado até uma pneumonia severa. Entretanto, alguns estudos apontam a possível relação da presente doença com o desenvolvimento de trombose venosa profunda e embolia pulmonar, devido ao intenso processo inflamatório e fenômenos hematológicos, especificamente a disfunção endotelial, estase sanguínea e ativação plaquetária (Roberto et al., 2020).

O SARS-CoV-2 rapidamente espalhou-se por vários países do mundo, indicando-o a alta taxa de transmissibilidade que o vírus possui (Magno et al., 2020). Sua transmissão acontece especialmente durante o contato próximo de pessoas, em menos de dois metros, através de gotículas respiratórias que são produzidas durante o espirro ou tosse de uma pessoa infectada. Outra possível forma para transmissão do SARS-CoV-2 é o contato com superfícies contaminadas seguido do toque em nariz, olhos ou boca. Estudos comprovam ainda que o vírus pode permanecer infeccioso e viável por horas no ar e dias em superfícies. A transmissão pode acontecer por pessoas assintomáticas, pré-sintomáticas e sintomáticas. Sendo sobretudo contagiosa por casos assintomáticos (Acter et al., 2020).

O SARS-CoV-2 é um vírus pertencente à família Coronaviridae, classificado como um novo Beta coronavírus (Chen, 2020). O mesmo possui quatro principais proteínas estruturais, tais como, proteína S (spike), proteína N (nucleocapsídeo), proteína $\mathrm{M}$ (membrana) e proteína $\mathrm{E}$ (envelope). Depois que acontece a infecção, a proteína $\mathrm{S}$ liga-se à enzima conversora de 
angiotensina II (ECA2), que é receptor na membrana celular e permite a entrada do vírus na célula. A infecção viral provoca uma desregulada resposta imunológica, além de ocasionar dano tecidual com trombose pulmonar no local que o vírus adentra a célula (Loureiro et al., 2020).

A infecção pelo SARS-CoV-2 está associada ao desenvolvimento de tromboembolismo pulmonar, em razão da interação entre macrófagos, polimorfonucleares, fatores de coagulação, plaquetas, relação com os níveis do Dímero-D e proteínas imunoefetores (Casella, 2020). São diversas as consequências prognosticas dos eventos trombóticos na COVID-19, entretanto, eles estão mais frequentemente ligados a lesões pulmonares, necessidade de ventilação mecânica e piora no prognóstico dos pacientes, levando-os para intenção na UTI (Fauvel et al., 2020).

O diagnóstico da COVID-19 é realizado por intermédio de exames laboratoriais que detectam o RNA viral ou anticorpos produzidos contra o vírus. Atualmente as técnicas de diagnósticos mais utilizados são: A reação em cadeia da polimerase de transcrição reversa em tempo real (RT-PCR), testes rápidos e sorológicos (Loeffelholz et al., 2020). Entretanto, diante das alterações hematológicas provocadas pela COVID-19, tem-se utilizados diferentes métodos de imagem em pacientes hospitalizados sintomáticos e com situações clínicas específicas. Sendo proibido a utilização de diagnóstico por imagem para confirmação inicial ou rastreio da COVID-19 (Loureiro et al., 2020).

No que tange o diagnóstico por imagem, a utilização da Radiologia (Raio-X), tem contribuído moderadamente, pois algumas alterações relevantes podem não se sobressair com essa técnica, porém há um predomínio elevado desse equipamento em hospitais e clínicas. Já a Tomografia Computadorizada (TC), dispõe de uma melhor demonstração de alterações associadas ao quadro clínico do paciente (Lima et al., 2020). Outro exame que está sendo amplamente utilizado para detecção de complicações decorrentes da COVID-19 é a Angiografia Pulmonar por Tomografia Computadorizada Multidetectores (TCMD), pois a mesma é considerada padrão ouro para diagnóstico de tromboembolismo pulmonar (costa et al., 2020).

Através deste artigo foi possível analisar criticamente a relação do SARS-CoV-2 com o tromboembolismo pulmonar, com o propósito de elucidar a necessidade de uma maior compreensão sobre as técnicas de diagnóstico por imagem que são utilizadas para confirmação do quadro clínico do paciente com COVID-19. O objetivo deste estudo foi apresentar a relação da infecção pelo SARS-CoV-2 com o tromboembolismo pulmonar, evidenciando assim, a importância dos exames de imagem no auxílio do diagnóstico desta patologia, enfatizando, as principais técnicas utilizadas, tais como: Radiologia de Tórax, Tomografia Computadorizada e Angiografia Pulmonar por Tomografia Computadorizada Multidetectores (TCMD).

\section{Metodologia}

O presente estudo trata-se de uma revisão integrativa de literatura, de abordagem descritiva e qualitativa, sendo assim esse tipo de estudo prever a utilização de grupamento de dados que podem ser analisados a partir de critérios numéricos e analíticos aplicado ao objetivo principal do estudo (Pereira et al., 2018). Dessa forma os indexados utilizados foram das bases de dados eletrônicas Centers for Disease Control and Prevention (CDC), Scientific Electronic Library Online (SciELO) e PubMed (MEDLINE), utilizando-se os termos: "COVID-19", "Exames de Imagem" "SARS-CoV-2" e "Tromboembolismo".

Foram encontrados 1.530 artigos relacionados à temática abordada, sendo selecionados apenas 40 artigos mediante os critérios de inclusão e exclusão. Os critérios para inclusão de artigos nesta revisão foram aplicados em artigos publicados no ano de 2020, nos idiomas espanhol, inglês e português e de maior impacto. Aplicaram-se os critérios de exclusão, onde foram descartados os artigos repetidos, indisponíveis na íntegra, que não se enquadravam no eixo temático e que não estavam no período de delimitação temporal. 
Figura 1. Passos da revisão integrativa de literatura.

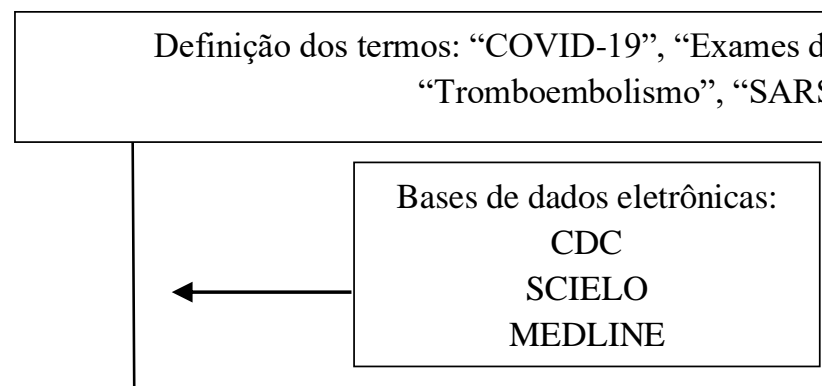

Artigos encontrados $(\mathrm{N}=1.530)$

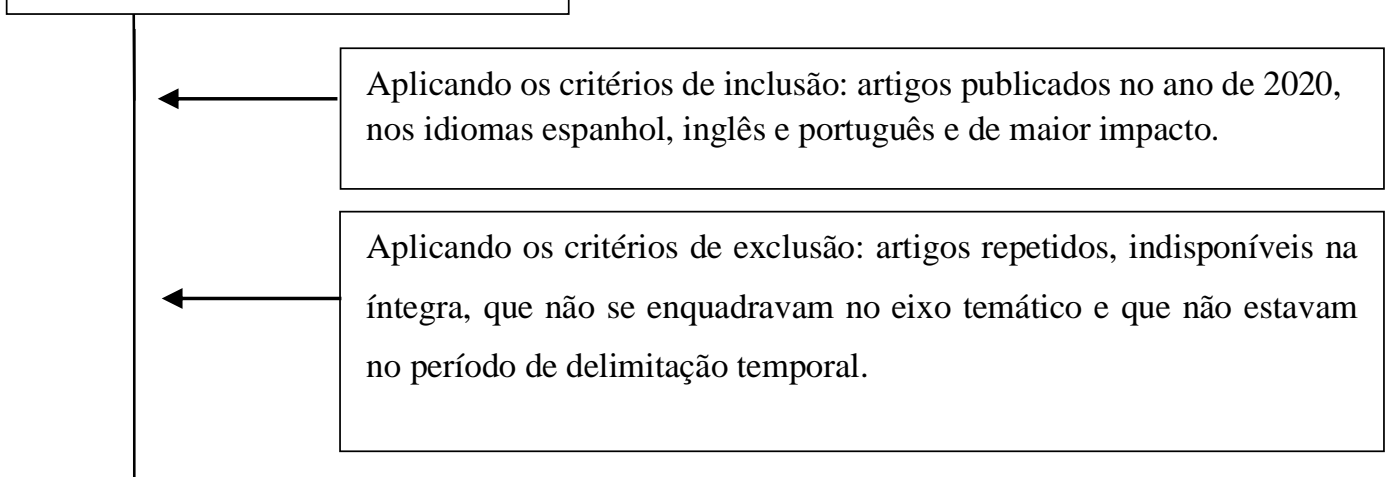

Artigos selecionados $(\mathrm{N}=40)$

Fonte: Autores (2021).

\section{Resultados e Discussão}

\subsection{Tromboembolismo pulmonar decorrente da infecção por SARS-CoV-2.}

Segundo Bikdeli et al. (2020), o SARS-CoV-2 é o vírus causador da doença do coronavírus (Covid-19), a qual é uma infecção viral respiratória que pode evoluir para doença trombótica ou formação de tromboembolismo, tanto em veias quanto em artérias, devido a uma excessiva inflamação, ativação plaquetária, disfunção endotelial e estase.

De acordo com Loureiro et al., (2020), os eventos trombóticos associados a COVID-19 são cada vez mais frequentes em pacientes, em razão do aumento exacerbado na produção de quimiocinas e citocinas pró-inflamatórias, que levam ao aumento da permeabilidade vascular, extravasamento de plasma e coagulação disfuncional.

O Tromboembolismo Pulmonar está correlacionado imunologicamente as respostas adaptativas do vírus, a causa do TEP agudo ainda não está totalmente esclarecida, porém pode estar relacionado com a lesão endotelial determinada pela ação viral (Nascimento et al., 2020). Resultante da oclusão da circulação arterial pulmonar o tromboembolismo pulmonar síndrome clínica e fisiopatológica elevam a pressão da artéria pulmonar, chegando em níveis não suportados ocasionando assim um óbito por dissociação eletromecânica (Amorim et al., 2020).

A infecção por SARS-CoV-2 está associada ao aumento de citocinas inflamatórias e distúrbios de coagulação com predisposição a formação de trombos. As células mononucleares interagem com as plaquetas ativadas e a cascata da coagulação, a qual estimulam as células inflamatórias por meio da ligação da trombina e do fator tecidual com receptores específicos ativados por protease e da ligação da fibrina ao receptor Toll-like4 (Figura 02). Posteriormente, a ativação das células inflamatórias resulta na liberação de citocinas pró-inflamatórias, que levam ao comprometimento das vias normais da coagulação e interrupção da fibrinólise (Nascimento et al., 2020). 
Figura 2. Processo inflamatório e trombótico na infecção por SARS-CoV-2.

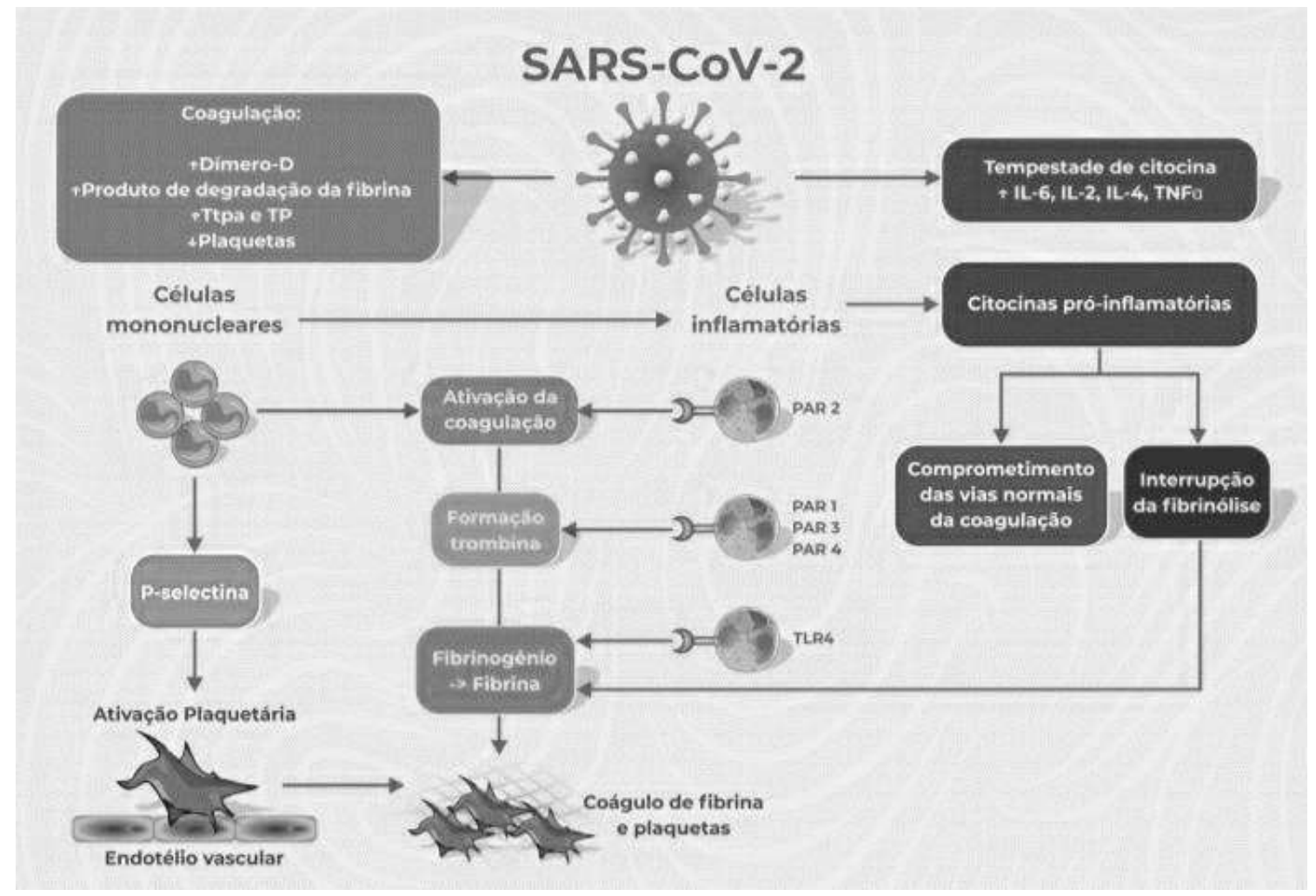

Fonte: Nascimento et al. (2020).

A infecção por SARS-CoV-2 provoca efeitos diretos ou os efeitos indiretos que podem causar o tromboembolismo, como por meio do agravo da doença ou por hipóxia que podem predispor os pacientes a eventos trombóticos, assim também como as terapias investigacionais para o tratamento da COVID-19, as quais podem ter interações medicamentosas adversas com agentes antiplaquetários e anticoagulantes, com a pandemia e a alocação de recursos ou recomendações de distanciamento social, pode afetar adversamente o cuidado de pacientes sem COVID-19, mas que apresentam eventos trombóticos (Bikdeli et al., 2020).

As anormalidades hemostáticas mais consistentes com COVID-19 incluem trombocitopenia leve e níveis aumentados de dímero $\mathrm{D}$, que estão associados a um risco maior de exigir ventilação mecânica, admissão à unidade de terapia intensiva (UTI) ou morte. No entanto, ainda não se sabe se essas alterações hemostáticas são um efeito específico do SARS-CoV-2 ou são uma consequência da tempestade de citocinas que precipita o aparecimento de Síndrome da Resposta Inflamatória Sistêmica (SIRS), como observado em outras doenças virais (Loureiro et al., 2020).

Para Bikdeli et al., (2020), na infecção pelo novo coronavírus, alguns pacientes apresentam uma produção excessiva dessas citocinas, aumentando a resposta inflamatória e a taxa de coagulação, causando a presença de microtrombos nos pulmões de alguns pacientes. Essas lesões nos pulmões podem deixar o paciente propenso a desenvolver trombose venosa profunda, provocando uma embolia pulmonar. Em contrapartida Leal et al., (2020), descreve que a presença de D-dímero representa uma sensibilidade de $100 \%$ e especificidade de $67 \%$ para embolia pulmonar, pois a fase da doença que acontece o desenvolvimento de síndrome do desconforto respiratório agudo e a piora do padrão radiológico é marcada pela produção exacerbada de D-dímero. 


\subsection{Exames de Imagem no Diagnóstico da COVID-19}

A infecção por SARS-CoV-2 manifestam sintomas mais evidentes em pacientes com testes moleculares reagentes, manifestações respiratórias e exames de imagem com resultados semelhantes com diagnóstico de pneumonia. Em sua maioria, os pacientes apresentam um bom prognóstico. Em idosos e pacientes que viviam com alguma comorbidade, como doenças renais e cardiovasculares, diabetes, a COVID-19 pode avançar para sua forma mais agressiva, com desconforto respiratório agudo (SDRA), e disfunção renal, hepática e cardíaca (Xavier, 2020).

Com a propagação mundial da COVID-19, os procedimentos de diagnóstico por meio de exames de imagem ganharam destaque, devido a incidência de doenças trombóticas ou pela formação de tromboembolismo em paciente acometidos pelo novo coronavírus. Dentre os exames de imagem que estão sendo utilizados, a Radiografia de tórax, TC de tórax e a Angiografia Pulmonar, apresentaram melhores resultados evidentes (Tabela 1) (Cbr, 2020).

Tabela 1. Exames de Imagem no Diagnóstico da COVID-19.

\begin{tabular}{|c|c|c|c|}
\hline AUTOR / ANO & TÍTULO & $\begin{array}{l}\text { EXAME DE } \\
\text { IMAGEM }\end{array}$ & DESCRIÇÃO \\
\hline Estevão (2020) & $\begin{array}{l}\text { COVID-19: artigo de } \\
\text { opinião }\end{array}$ & $\begin{array}{l}\text { Radiografia de } \\
\text { tórax } \\
\text { (Raio-X) }\end{array}$ & $\begin{array}{l}\text { O Raio-X apresenta desempenho limitado, em razão da } \\
\text { sua baixa sensibilidade e especificidade. Através do Raio- } \\
\text { X é possível identificar consolidação compatíveis com } \\
\text { pneumonia, apesar de frequentes em casos graves da } \\
\text { COVID-19, não são critérios específicos da infecção por } \\
\text { SARS-CoV-2. }\end{array}$ \\
\hline $\begin{array}{l}\text { Farias et al., } \\
\qquad(2020)\end{array}$ & $\begin{array}{l}\text { Alterações } \\
\text { tomográficas torácicas } \\
\text { em pacientes } \\
\text { sintomáticos } \\
\text { respiratórios com a } \\
\text { COVID-19 }\end{array}$ & $\begin{array}{l}\text { Tomografia } \\
\text { Computadorizada } \\
\text { (TC) }\end{array}$ & $\begin{array}{l}\text { Tem-se a tomografia computadorizada como um método } \\
\text { bastante sensível que evidencia as alterações decorrentes } \\
\text { do acometimento pulmonar ocasionado pelo SARS-CoV- } \\
\text { 2. A mesma pode ser utilizada como forma de } \\
\text { diagnóstico auxiliar em pacientes de COVID-19, que } \\
\text { estejam em situações clínicas especificas. }\end{array}$ \\
\hline $\begin{array}{l}\text { Dueñas et al., } \\
\qquad(2020)\end{array}$ & $\begin{array}{c}\text { Pulmones azules en } \\
\text { pacientes COVID-19: } \\
\text { un passo más allá del } \\
\text { diagnóstico de } \\
\text { tromboembolismo } \\
\text { pulmonar mediante } \\
\text { TCMD con mapa de } \\
\text { yodo }\end{array}$ & $\begin{array}{l}\text { Angiografia } \\
\text { Pulmonar por } \\
\text { Tomografia } \\
\text { Computadorizada } \\
\text { Multidetectores } \\
\text { (TCMD) }\end{array}$ & $\begin{array}{l}\text { A utilização da angiografia pulmonar TCMD possibilita } \\
\text { dentre as técnicas de imagem, uma maior sensibilidade } \\
\text { para detecção de tromboembolismo pulmonar. }\end{array}$ \\
\hline
\end{tabular}

Fonte: Autores (2021).

A Radiografia de tórax é um exame prático e simples, este método é mais indicado em pacientes acamados, onde é feito um maior monitoramento de progressão da doença, avaliando os tubos traqueais e as linhas de infusão medicamentosa, descartando possíveis complicações como: pneumotórax, pneumomediastino e enfisema subcutâneo (Estevão, 2020). Contudo, mesmo com a sua facilidade de desempenho, o exame apresenta baixa sensibilidade, variando entre 30-69\%, com exames normais em formas mais leves da doença. Em exames que aparecem alterações, os principais achados são consolidações (3647\%) e imagens de baixa densidade e opacidade (20-33\%), frequente predomínio basal periférico. As apresentações de derrame pleural são mais incomuns, representando apenas em 3\% dos pacientes em um estudo realizado por Wong et al., (2020).

Para Kim et al., (2020), a TC de tórax é considerada o método mais indicado em pacientes com suspeita de COVID19. Este exame apresenta uma sensibilidade de $94 \%$ e especificidade de $37 \%$. O que demonstra em regiões menos prevalentes da doença, a utilização da TC de tórax é associada a maiores números de falso-positivos, o que poderia aumentar os custos 
médicos e exames adicionais. Salehi (2020) descreve que geralmente a TC de tórax é realizado sem a utilização de contraste intravenoso, espessura de corte de 1,0mm e se possível, em baixa dose de radiação. O uso do contraste se faz necessário apenas quando houver complicações por tromboembolismo pulmonar. Os achados mais evidentes na TC de tórax são opacidades em vidro fosco, pavimentações em mosaico, consolidações, opacidades reticulares, linhas subpleurais, sinal do halo invertido e espessamento pleural.

Durante várias décadas a angiografia pulmonar por tomografia computadorizada multidetectores (TCMD) é escolhida como a técnica de imagem utilizada para descartar o tromboembolismo pulmonar. Entretanto, pequenos defeitos de enchimento nos ramos arteriais distais, de pequeno calibre, podem passar despercebidos. Atualmente, utiliza-se a angiografia pulmonar de TCMD com mapeamento de iodo em pacientes com COVID-19, devido a sensibilidade que essa técnica apresenta para detecção de tromboembolismo pulmonar, bem como, o mapa de iodo é considerado um indicador de microtrombose, permitindo então o início rápido do tratamento com anticoagulantes e, assim reduzindo a taxa de mortalidade dessa patologia em pacientes com COVID-19 (Dueñas et al., 2020).

Tabela 2. Achados sobre a associação de embolia pulmonar e COVID-19.

\begin{tabular}{|c|c|c|c|}
\hline AUTOR / ANO & TÍTULO & $\begin{array}{l}\text { TIPOS DE } \\
\text { ESTUDO }\end{array}$ & DESCRIÇÃO \\
\hline $\begin{array}{c}\text { Leonard-Lorant } \\
\text { et al., (2020) }\end{array}$ & $\begin{array}{l}\text { Acute Pulmonary } \\
\text { Embolism in Patients } \\
\text { with COVID-19 at CT } \\
\text { Angiography and } \\
\text { Relationship to D- } \\
\text { Dimer Levels. }\end{array}$ & $\begin{array}{c}\text { Estudo } \\
\text { retrospectivo. }\end{array}$ & $\begin{array}{l}\text { Demonstrou que de } 106 \text { angiografias pulmonares por } \\
\text { TC realizadas para pacientes com COVID-19 durante } \\
\text { um período de um mês em um centro de atendimento } \\
\text { terciário; } 32 / 106 \text { (30\%) dos pacientes tiveram embolia } \\
\text { pulmonar aguda. Essa taxa de embolia pulmonar é } \\
\text { maior do que a geralmente encontrada em pacientes } \\
\text { criticamente enfermos sem infecção por COVID-19 } \\
(1,3 \%) \text { ou em pacientes de pronto-socorro ( } 3 \text { a } 10 \%) \text {. }\end{array}$ \\
\hline $\begin{array}{l}\text { Fauvel et al., } \\
\qquad(2020)\end{array}$ & $\begin{array}{l}\text { Pulmonary embolism in } \\
\text { COVID-19 patients: a } \\
\text { French multicentre } \\
\text { cohort stugy. }\end{array}$ & $\begin{array}{l}\text { Estudo } \\
\text { observacional } \\
\text { multicêntrico } \\
\text { retrospectivo. }\end{array}$ & $\begin{array}{l}\text { Foram incluídos pacientes consecutivos hospitalizados } \\
\text { por COVID-19. Pacientes sem diagnóstico de EP } \\
\text { comprovado por angiotomografia pulmonar por } \\
\text { tomografia computadorizada (CTPA) e aqueles que } \\
\text { foram internados diretamente em uma unidade de } \\
\text { terapia intensiva (UTI) foram excluídos. }\end{array}$ \\
\hline $\begin{array}{l}\text { Lang et al., } \\
\text { (2020) }\end{array}$ & $\begin{array}{l}\text { Pulmonary Vascular } \\
\text { Manifestations of } \\
\text { COVID-19 Pneumonia. }\end{array}$ & $\begin{array}{c}\text { Estudo } \\
\text { retrospectivo }\end{array}$ & $\begin{array}{l}48 \text { pacientes com infecção por COVID- } 19 \text { confirmada } \\
\text { por RT-PCR que foram submetidos à angiografia } \\
\text { pulmonar por TC entre 22/03/20 e 5/4/20 em nosso } \\
\text { grande sistema de saúde urbano foram incluídos. Os } \\
\text { dados demográficos e clínicos dos pacientes foram } \\
\text { coletados por meio do sistema de prontuário eletrônico. }\end{array}$ \\
\hline
\end{tabular}

Fonte: Autores (2021).

Em um estudo realizado por Chamorro (2020), foram realizadas 492 angiografia-TCs pulmonares, onde foram incluídos 342 (69,9\%) pacientes com COVID-19 e 147 (30,1\%) pacientes sem infecção. A incidência de tromboembolismo pulmonar foi de $26 \%$ positivo e 16,3\% negativo, ambos resultados para o grupo com COVID-19, possuindo um risco relativo a 1,6 vezes maior para desenvolvimento dessa patologia em pacientes com COVID-19. Os resultados obtidos por Chamorro apontaram que 89 pacientes com COVID-19 e 24 pacientes sem COVID-19 apresentaram tromboembolismo pulmonar.

\subsection{Achados Tomográficos em COVID-19}

A tomografia computadorizada (TC) de alta resolução, mostra-se útil no contexto atual de pandemia, especialmente nos casos mais graves, na avaliação da extensão da doença, em possíveis diagnósticos diferenciais e na pesquisa de complicações (Tabela 2). Ainda que não patognomônicas e que possam se sobrepor aos achados de outras infecções virais, algumas 
alterações tomográficas da COVID-19 têm características que as destacam daquelas vistas em outras infecções, muitas vezes sendo bastante sugestivas desta infecção em particular (Farias et al., 2020).

Tabela 3. Achados Tomográficos na COVID-19.

\begin{tabular}{|c|c|c|c|}
\hline AUTOR / ANO & TÍTULO & $\begin{array}{c}\text { TIPOS DE } \\
\text { ACHADOS }\end{array}$ & DESCRIÇÃO \\
\hline $\begin{array}{l}\text { Farias et al., } \\
\qquad(2020)\end{array}$ & $\begin{array}{l}\text { Alterações tomográficas } \\
\text { torácicas em pacientes } \\
\text { sintomáticos } \\
\text { respiratórios com a } \\
\text { COVID-19. }\end{array}$ & $\begin{array}{l}\text { Padrão } \\
\text { Reticular }\end{array}$ & $\begin{array}{c}\text { Caracteriza-se pelo espessamento dos septos } \\
\text { interlobulares e intralobulares, manifestando-se como } \\
\text { opacidades lineares. Entretanto, não é um achado } \\
\text { comum da fase inicial da COVID-19, porém é } \\
\text { observado em menos de } 22 \% \text { dos casos, principalmente } \\
\text { na fase tardia da doença. }\end{array}$ \\
\hline $\begin{array}{l}\text { Farias et al., } \\
\qquad(2020)\end{array}$ & $\begin{array}{l}\text { Alterações tomográficas } \\
\text { torácicas em pacientes } \\
\text { sintomáticos } \\
\text { respiratórios com a } \\
\text { COVID-19. }\end{array}$ & $\begin{array}{l}\text { Pavimentoso } \\
\text { em Mosaico }\end{array}$ & $\begin{array}{l}\text { Refere-se à superposição das opacidades em vidro } \\
\text { fosco e o espaçamento dos septos interlobulares e } \\
\text { intralobulares, com sua interfase bem delimitada como } \\
\text { parênquima pulmonar normal adjacente, refletindo o } \\
\text { acometimento alveolar e intersticial. }\end{array}$ \\
\hline $\begin{array}{l}\text { Rosa et al., } \\
\text { (2020) }\end{array}$ & $\begin{array}{l}\text { Achados da COVID-19 } \\
\text { identificados na } \\
\text { tomografia } \\
\text { computadorizada de } \\
\text { tórax: ensaio pictórico. }\end{array}$ & Consolidação & $\begin{array}{l}\text { Refere-se a substituição do ar do espaço alveolar por } \\
\text { células, tecidos ou fluidos patológicos que obscurecem } \\
\text { os vasos locais e as margens dos brônquios. Sendo a } \\
\text { segunda alteração mais comumente encontrada na } \\
\text { pneumonia causada pela COVID-19, após as } \\
\text { opacidades em vidro fosco. }\end{array}$ \\
\hline $\begin{array}{l}\text { Rosa et al., } \\
(\mathbf{2 0 2 0})\end{array}$ & $\begin{array}{l}\text { Achados da COVID-19 } \\
\text { identificados na } \\
\text { tomografia } \\
\text { computadorizada de } \\
\text { tórax: ensaio pictórico. }\end{array}$ & $\begin{array}{l}\text { Múltiplas } \\
\text { Opacidades } \\
\text { em Vidro } \\
\text { Fosco }\end{array}$ & $\begin{array}{c}\text { Descrita com morfologia arredondada, predominando } \\
\text { na periferia dos lobos pulmonares e em suas regiões } \\
\text { posteriores, frequentemente nas bases. O acometimento } \\
\text { é, na maioria das vezes, bilateral e multilobar. }\end{array}$ \\
\hline
\end{tabular}

Fonte: Autores (2021).

A Tomografia Computadorizada é um método de diagnóstico essencial e pode auxiliar na avaliação da extensão da COVID-19, devido as possíveis complicações e na confirmação de diagnósticos alternativos, porém para realização do diagnóstico da COVID-19 recomenda-se a utilização do teste laboratorial "Reação em Cadeia da Polimerase de Transcrição Reversa" (RT-PCR), sendo recomendada a utilização da TC quando o paciente estiver hospitalizado e apresentar sintomas específicos, sendo necessário a confirmação do acometimento pulmonar através da TC (Rosa et al., 2020).

\section{Conclusão}

SARS-CoV-2 é o vírus responsável por causar COVID-19, a qual é uma doença que acomete o trato respiratório inferior e superior. A infecção por SARS-CoV-2 se apresenta com um fenótipo variável, sendo frequentes os relatos de complicações devido as alterações hematológicas que predispõem a presença de um estado pró-trombótico e, a formação de tromboembolismo pulmonar, diante dessas alterações faz-se necessário a utilização dos exames por imagem, sendo eles imprescindíveis para a confirmação do diagnóstico. Os exames de imagem podem auxiliar na avaliação da extensão de doença, das possíveis complicações e na determinação de diagnósticos alternativos.

Sugerem-se a aplicação de mais estudos aprofundados devido à escassez sobre o assunto, cujo, engloba as duas patologias (SARS-CoV-2 e Tromboembolismo Pulmonar), conforme o objetivo seja a inserção concreta de possíveis necessidades da população. Os resultados entre os exames de imagem intensificam com maior avaliação desses estudos, ou seja, considera-se padrão a forma de avaliação das demais estruturas anatômicas do paciente, possibilitando uma melhor 
interpretação. Incluindo que alguns desses tem um custo benefício, facilitando a função. Esse tipo de diagnóstico também pode avaliar o desfecho clínico determinante da patologia encontrada.

\section{Referências}

Acter, T., Uddin, N., Das, J., Akhter, A., Choudhury, T. R., \& Kim, S. (2020). Evolution of severe acute respiratory syndrome coronavirus 2 (SARS-CoV-2) as coronavirus disease 2019 (COVID-19) pandemic: A global health emergency. Science of the Total Environment, 138996.

Amorim, D. S., Lima, F. L. O., \& Costa, E. A. S. (2020). Tromboembolismo Pulmonar Em Covid-19. Hematology, Transfusion and Cell Therapy, 42, 562.

Beraldo, G. L., Fonseca, E. K. U. N., Yokoo, P., Matos, M. J. R. D., Rosa, M. E. E., Silva, M. M. A., \& Ishikawa, W. Y. (2020). Pneumonia pelo novo coronavírus e tromboembolismo pulmonar agudo: casualidade ou causalidade? Einstein (São Paulo), 18.

de Carvalho Bezerra, L., Pires, G. M., de Oliveira Sousa, H. F., de Albuquerque, L. T. C., de Morais, M. G. G., \& Pinheiro, R. X. M. (2020). Radiological Images of Pulmonary Thromboembolism in a Patient with the Diagnosis of Covid-19: A Case Report. Amadeus International Multidisciplinary Journal, 4(8), 98-101.

Bikdeli, B., Madhavan, M. V., Jimenez, D., Chuich, T., Dreyfus, I., Driggin, E., \& Lip, G. Y. (2020). COVID-19 and thrombotic or thromboembolic disease: implications for prevention, antithrombotic therapy, and follow-up: JACC state-of-the-art review. Journal of the American college of cardiology, 75(23), 2950-2973

Casella, I. B. (2020). Physiopathology of SARS-CoV-2-infection-associated thrombosis. Jornal Vascular Brasileiro, 19.

da Silveira Cespedes, M., \& Souza, J. C. SARS-CoV-2: uma revisão para o clínico.

Chamorro, E. M., Ostolaza, T. R., Núñez, M. P., Nacenta, S. B., Rodríguez-Guerra, C. C. C., \& Sanz, L. I. (2021). Tromboembolismo pulmonar en pacientes con COVID-19: estudio de prevalencia en un hospital terciario. Radiología, 63(1), 13-21.

Chate, R. C., Fonseca, E. K. U. N., Passos, R. B. D., Teles, G. B. D. S., Shoji, H., \& Szarf, G. (2020). Presentation of pulmonary infection on CT in COVID19: initial experience in Brazil. Jornal Brasileiro de Pneumologia, 46(2).

Chen, J. (2020). Pathogenicity and transmissibility of 2019-nCoV-a quick overview and comparison with other emerging viruses. Microbes and infection, 22(2), 69-71.

Colégio Brasileiro de Radiologia e Diagnóstico por Imagem (CBR). (2020). Recomendações de uso de métodos de imagem para pacientes suspeitos de infecção pelo COVID-19.

Costa, I. B. S. D. S., Rochitte, C. E., Campos, C. M., Barberato, S. H., Oliveira, G. M. M. D., Lopes, M. A. C. Q., \& Hajjar, L. A. (2020). Imagem Cardiovascular e Procedimentos Intervencionistas em Pacientes com Infecção pelo Novo Coronavírus. Arquivos Brasileiros de Cardiologia, 115(1), 111-126.

Danzi, G. B., Loffi, M., Galeazzi, G., \& Gherbesi, E. (2020). Acute pulmonary embolism and COVID-19 pneumonia: a random association? European heart journal, 41(19), 1858-1858.

Pérez Dueñas, V., Allona Krauel, M., Agrela Rojas, E., Ramírez Prieto, M. T., Díez Izquierdo, L., López de la Guardia, U., \& Torres Sánchez, I. (2021). Pulmones azules en pacientes COVID-19: un paso más allá del diagnóstico de tromboembolismo pulmonar mediante TCMD con mapa de yodo. Arch. bronconeumol.(Ed. impr.), 35-46.

Estevão, A. (2020). COVID-19. Acta Radiológica Portuguesa, 32(1), 5-6.

Farias, L. D. P. G. D., Strabelli, D. G., Fonseca, E. K. U. N., Loureiro, B. M. C., Nomura, C. H., \& Sawamura, M. V. Y. (2020). Alterações tomográficas torácicas em pacientes sintomáticos respiratórios com a COVID-19. Radiologia Brasileira, 53(4), 255-261.

Fauvel, C., Weizman, O., Trimaille, A., Mika, D., Pommier, T., Pace, N., \& Critical Covid-19 France Investigators. (2020). Pulmonary embolism in COVID19 patients: a French multicentre cohort study. European heart journal, 41(32), 3058-3068.

Araujo-Filho, J. D. A. B., Sawamura, M. V. Y., Costa, A. N., Cerri, G. G., \& Nomura, C. H. (2020). Pneumonia por COVID-19: qual o papel da imagem no diagnóstico?. Jornal Brasileiro de Pneumologia, 46(2).

Guo, T., Fan, Y., Chen, M., Wu, X., Zhang, L., He, T., \& Lu, Z. (2020). Cardiovascular implications of fatal outcomes of patients with coronavirus disease 2019 (COVID-19). JAMA cardiology, 5(7), 811-818.

Jasinowodolinski, D., Filisbino, M. M., \& Baldi, B. G. (2020). Pneumonia por COVID-19: um fator de risco para tromboembolismo pulmonar?. Jornal Brasileiro de Pneumologia, 46(4), e20200168-e20200168.

Kim, H., Hong, H., \& Yoon, S. H. (2020). Diagnostic performance of CT and reverse transcriptase polymerase chain reaction for coronavirus disease 2019: a meta-analysis. Radiology, 296(3), E145-E155.

Lang, M., Som, A., Carey, D., Reid, N., Mendoza, D. P., Flores, E. J., \& Little, B. P. (2020). Pulmonary vascular manifestations of COVID-19 pneumonia. Radiology: Cardiothoracic Imaging, 2(3), e200277.

Soeiro, A. D. M., Leal, T. D. C. A. T., Pereira, M. D. P., Lima, E. G., Figueiredo, A. C. B. D. S., Petriz, J. L. F., \& Serrano Jr, C. V. (2020). Position Statement on the Use of Antiplatelet Agents and Anticoagulants in Patients Infected with the New Coronavirus (COVID-19)-2020. Arquivos Brasileiros de Cardiologia, 115(2), 292-301. 
Research, Society and Development, v. 10, n. 5, e36110514989, 2021

(CC BY 4.0) | ISSN 2525-3409 | DOI: http://dx.doi.org/10.33448/rsd-v10i5.14989

Leonard-Lorant, I., Delabranche, X., Severac, F., Helms, J., Pauzet, C., Collange, O., \& Ohana, M. (2020). Acute pulmonary embolism in patients with COVID-19 at CT angiography and relationship to D-dimer levels. Radiology, 296(3), E189-E191.

Lima, C. M. A. D. O. (2020). Informações sobre o novo coronavírus (COVID-19). Radiologia Brasileira, 53(2), V-VI.

Lima, F. L. O., Gomes, L. N. L., dos Santos, C. S. C., \& de Oliveira, G. A. L. (2020). Diagnóstico da COVID-19: importância dos testes laboratoriais e dos exames de imagem. Research, Society and Development, 9(9), e259997162-e259997162.

Lippi, G., Plebani, M., \& Henry, B. M. (2020). Thrombocytopenia is associated with severe coronavirus disease 2019 (COVID-19) infections: a metaanalysis. Clinica chimica acta, 506, 145-148.

Loeffelholz, M. J., \& Tang, Y. W. (2020). Laboratory diagnosis of emerging human coronavirus infections-the state of the art. Emerging microbes \& infections, 9(1), 747-756.

Loureiro, Camila Melo Coelho et al. (2020). Alterações Pulmonares na COVID-19. Rev. Cient. Pages 89-99. Obtido em: file://C:/Users/User/Downloads/175Texto\%20do\%20artigo-646-1-10-20200827\%20(1).pdf.

Magno, L., Rossi, T, A, Mendonça-Lima, F, W, D., Santos, C, C, D., Campos, G, B., Marques, L, M., \& Dourado, I. (2020). Desafios e propostas para expandir os testes e diagnósticos COVID-19 no Brasil. Ciencia \& saude coletiva, 25 , 3355-3364.

Meirelles, G. D. S. P. (2020). COVID-19: a brief update for radiologists. Radiologia Brasileira, 53(5), 320-328.

Nascimento, J. H. P., Gomes, B. F. D. O., Carmo Júnior, P. R. D., Petriz, J. L. F., Rizk, S. I., Costa, I. B. S. D. S., \& Oliveira, G. M. M. D. (2020). COVID-19 e estado de hipercoagulabilidade: uma nova perspectiva terapêutica. Arquivos Brasileiros de Cardiologia, 114(5), 829-833.

Passos, H. D., Alves, M. C., Baumworcel, L., Vieira, J. P. C., Garcez, J. D. S., \& Sousa, A. C. S. (2020). SARS-Cov-2 Infection and Pulmonary Thromboembolism-The Prothrombotic State in COVID-19. Arquivos brasileiros de cardiologia, 115(1), 142-145.

Pereira, A. S., Shitsuka, D. M., Parreira, F. J., \& Shitsuka, R. (2018). Metodologia da pesquisa científica.

Roberto, Gabriel Antônio et al. (2020). COVID-19 e eventos tromboemmbólicos. ULAKES Jounal of Medicine. Pages 50-59.

Rosa, M. E. E., Matos, M. J. R. D., Furtado, R. S. O. D. P., Brito, V. M., Amaral, L. T. W., Beraldo, G. L., \& Funari, M. B. D. G. (2020). COVID-19 findings identified in chest computed tomography: a pictorial essay. Einstein (São Paulo), 18.

Salehi, S., Abedi, A., Balakrishnan, S., \& Gholamrezanezhad, A. (2020). Coronavirus disease 2019 (COVID-19): a systematic review of imaging findings in 919 patients. American Journal of Roentgenology, 215(1), 87-93.

Wong, H. Y. F., Lam, H. Y. S., Fong, A. H. T., Leung, S. T., Chin, T. W. Y., Lo, C. S. Y., \& Ng, M. Y. (2020). Frequency and distribution of chest radiographic findings in patients positive for COVID-19. Radiology, 296(2), E72-E78.

Xavier, A. R., Silva, J. S., Almeida, J. P. C., Conceição, J. F. F., Lacerda, G. S., \& Kanaan, S. (2020). COVID-19: clinical and laboratory manifestations in novel coronavirus infection. Jornal Brasileiro de Patologia e Medicina Laboratorial, 56.

Yang, W., Cao, Q., Qin, L. E., Wang, X., Cheng, Z., Pan, A., \& Yan, F. (2020). Clinical characteristics and imaging manifestations of the 2019 novel coronavirus disease (COVID-19): a multi-center study in Wenzhou city, Zhejiang, China. Journal of Infection, 80(4), 388-393.

Zuo, Y., Zuo, M., \& Yalavarthi, S. (2020). Neutrophil extracellular traps and thrombosis in COVID-19. medRxiv. Preprint, 5. 